\title{
Radar signal design problem with neural network processing
}

\author{
C KRISHNAMOHAN RAO ${ }^{\mathrm{a}}$ and P S MOHARIR ${ }^{\mathrm{b}}$
}

${ }^{a}$ VNR Vignana Jyothi Institute of Engineering and Technology, Jawaharlal Nehru Technological University, Hyderabad 500 072, India

Present address: Department of Electrical \& Computer Engineering, University of Missouri - Rolla, Rolla, MO 65409, USA

${ }^{\mathrm{b}}$ National Geophysical Research Institute, Uppal Road, Hyderabad 500 007, India

e-mail:ckm_rao@hotmail.com; pmoharir@rediffmail.com

MS received 21 September 1999

\begin{abstract}
Binary and ternary sequences with peaky autocorrelation, measured in terms of high discrimination and merit factor have been searched earlier, using optimization techniques. It is shown that the use of neural network processing of the return signal is much more advantageous. It opens up a new signal design problem, which is solved by an optimization technique called Hamming scan, for both binary and ternary sequences.
\end{abstract}

Keywords. Radar signal design; neural network processing; peaky autocorrelation; high discrimination; merit facotr.

\section{Introduction}

This paper deals with pulse compression sequences usable in radar. They need peaky autocorrelation and as small sidelobe amplitudes as possible. The satisfaction of these criteria is normally assessed on the basis of discrimination and merit factor (Golay 1977). Binary (alphabet: $-1,1$ ) and ternary (alphabet: $-1,0,1)$ sequences with high discrimination and merit factor have been looked for earlier using optimization techniques, including the Hamming scan algorithms (Moharir et al 1996-1998; Bernasconi 1977). The results were much better than those obtained by earlier classical search techniques, both in terms of merit factor and discrimination and the lengths of sequences up to which such results were obtained. With expendable computer time, there was still some dissatisfaction, which related to steady decrease in merit factor on the average with increase in the length of the sequence and to slow increase in the discrimination in the same sense (Moharir et al 1998). This led to investigation of the beneficial role that neural 
networks could play. Kwan \& Lee (1993) proposed this new approach for pulse compression using neural networks. The 13-element Barker code, and pseudo-noise (PN) sequences of lengths 15,31 and 63 were taken as the sequences to be used. Neural network target output was defined as the length of the sequence, when it was the input, and zero when any shifted version of the sequence was the input. The neural network was trained using the backpropagation learning algorithm. Deergha Rao \& Sridhar (1995) proposed the use of the learning algorithm based on the extended Kalman filtering, as that has faster convergence speed than the backpropagation algorithm. The results obtained were superior to those obtained with the backpropagation algorithm.

\section{Neural network processing}

Autocorrelation function of a good pulse compression sequence has a strong main peak. It is equal to the length $N$ of the sequence for binary sequences and equals the number $N_{n z}$ of nonzero elements in the sequence for ternary sequences. The sidelobes are autocorrelation values at nonzero lags. These cannot be zero for all lags. But now consider an autocorrelator as a block which gives a single output, when the sequence or some shifted (or translated) version of it is the input. Let the output be the value of the autocorrelation at the corresponding lag. Next, consider the autocorrelator block as being replaced by a radial basis network, which is used in the present work, or any other neural network. The autocorrelator is a device that has no parameters of its own. But the radial basis network has its own parameters. So let its target output be defined as the main peak of the autocorrelation when the input is the entire unshifted (zero-lag) sequence and zero for all shifted versions (lags $= \pm(N-1), \pm(-2), \ldots, \pm 1)$ of the sequences as the input. The output of the radial basis network is not the auto correlation. Let it be called the translation signature. This is to be adjusted to have sidelobes as weak as possible. The autocorrelation of a sequence is entirely its property and the discrete autocorrelator merely calculates it. The translation signature is a joint property of the sequence and the radial basis network. Hence the scope for an improvement in the performance of a sequence when the crosscorrelator or the matched filter is replaced by the radial basis neural network processor. The measures of discrimination and merit factor are defined in terms of the translation signature provided by the trained neural network. The improvement achieved is discussed later.

An important point must be made here. The cross-correlation that is calculated in radar practice is an analog operation. The two inputs to it are the return waveform and the transmitted waveform. That is, no detection after reception is needed. When the neural network is used, the input to it has to be a discrete sequence, as the former is a discrete device. Therefore, detection after reception and before neural network processing would be needed. This change in the technology is justified in terms of the enormous improvement in sidelobe reduction.

To establish firmly the advantage obtainable by neural network processing, and to understand the effect of the length of the sequence, good binary and ternary sequences of many lengths were taken and their merit factors and discriminations were reassessed in terms of the translation signature provided by the trained radial basis neural network. These neural networks were proposed by Chen et al (1991), and benchmarking reported (Demuth \& Beale 1997) was the guiding factor in choosing them. Golay (1977) had listed 
a

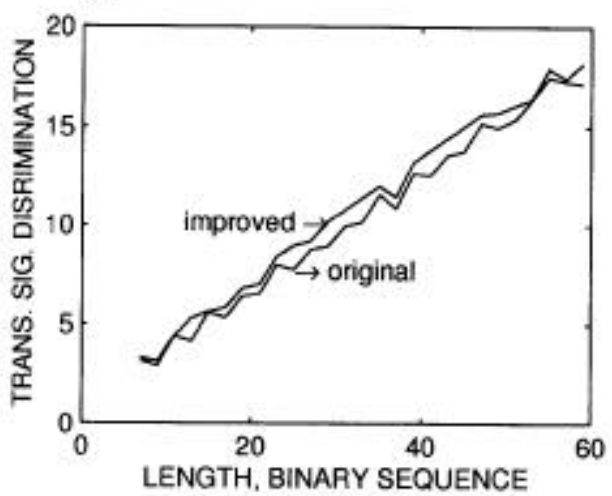

C

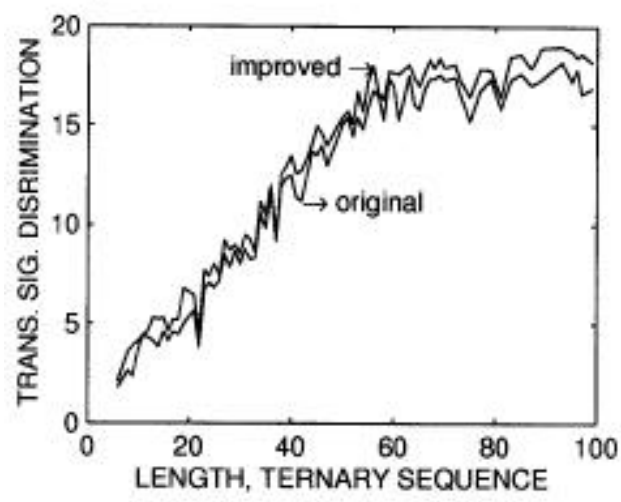

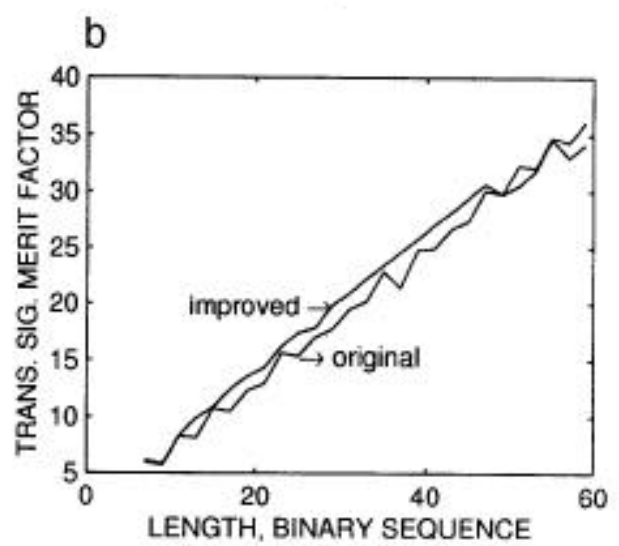

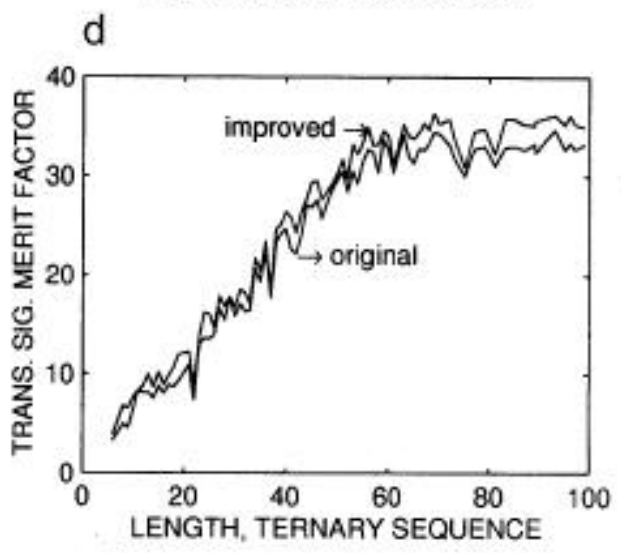

Figure 1. (a) \& (b) Plots pertain to binary sequences while (c) \& (d) to ternary sequences. (a) \& (c) show the discrimination based on the translation signature returned by the trained radial basis neural network. (b) \& (d) show merit factor of the translation signature. In every plot, the lower curve pertains to the original sequence chosen for the best merit factor for the binary case, and best discrimination for the ternary case. The upper curves give the parameters of the solution of the neural network signal design problem.

binary sequences with best possible merit factors up to a length of 59. Discrimination and merit factors of the translation signature provided by the radial basis network were calculated. The parameters of the trained radial basis network chosen were spread $=1$ and maximum number of neurons in the hidden layer $=3$. Weights and biases which gave the best sidelobe suppression are stored for future use in a separate data file for every sequence. Similar work was done for ternary sequences up to the length of 99, listed by Moharir et al (1998) for maximum discrimination. The results are shown in figure 1 by the lower curves. The best merit factors for the binary and ternary sequences are 14.08 (length 13) and 20.06 (length 23), respectively. Below a length of 100, the ternary sequence gives the best discrimination of 20 at the length of 24 . The advantage of neural network processing over these results is thus enormous. 


\section{New signal design problem}

The use of neural network processing naturally introduces a new signal design problem. Why should we merely determine the merit factor and discrimination of the translation signature provided by the trained neural network, for binary and ternary sequences, which were good on the basis of classical measures of merit factor and discrimination? A more justifiable approach would be to obtain $a b$ initio binary and ternary sequences with the best merit factor and discrimination for the translation signature provided by the trained neural network. This signal design problem is solved. Many modern global optimization algorithms employ probabilistic searches that are computationally prohibitive. A more efficient algorithm, viz. Hamming scan (Moharir et al 1996-1998) is employed, in which the entirely probabilistic mechanism is replaced by a locally complete search. Hamming scan involves a search among all the first-order Hamming neighbours of the sequence, and moving to the best among those neighbours. This procedure is recursively used as long as it works. The improvement in the measures of interest is shown by the upper curves in figure 1. It is obvious that sequences that are best for classical correlation processing are not the best for neural network processing.

\section{Conclusions}

The use of neural networks leads to a manifold increase in the measures of interest, viz. discrimination and merit factor. The search for the best sequences is a new signal design problem, once we decide to use neural network processing at the receiver. Discrimination defined on the autocorrelation increases slowly on an average, as the length of the sequence increases. The merit factor, on the other hand, decreases under similar conditions. That makes the choice of a proper criterion somewhat difficult. When discrimination and merit factor are defined on the basis of the translation signature of a trained radial basis neural network, both of them improve on the average with increasing length. Some reduction in the average slope can be seen in figure 1. It may be countered by a fresh choice of the two chosen parameters of the radial basis network. These parameters are held constant for all lengths, just to make comparisons fairer. With discrimination and merit factor defined on the basis of autocorrelation, ternary sequences are shown to yield considerably higher values. When discrimination and merit factor are defined on the basis of translation signature of a trained radial basis neural network, this advantage of the ternary sequences disappears. This strengthens the starting motivation of this work that a change in the method of processing at the receiver suggests a new signal design problem, by proving that even the gross conclusions drawn earlier from the solution of the previous signal design problem need revision.

\section{References}

Bernasconi J 1987 Low autocorrelation binary sequences: statistical mechanics and configuration space analysis. J. Phys. 48: 559-567

Chen S, Cowan C F N, Grant P M 1991 Orthogonal least squares learning algorithm for radial basis function networks. IEEE Trans. Neural Networks. NN-2: 302-309

Deergha Rao K, Sridhar G 1995 Improving performance in pulse radar detection using neural networks. IEEE Trans. Aerosp. Electron. Syst. AES-31: 1193-1198 
Demuth H, Beale M 1997 MATLAB neural network toolbox, Users guide, Version 2. The Math Works Inc., Natick, pp 6.1 to 6.13

Golay M J E 1977 Sieves for low autocorrelation binary sequences. IEEE Trans. Inf. Theory IT-23: 43-51

Kwan H K, Lee C K 1993 A neural network approach to pulse radar detection. IEEE Trans. Aerosp. Electron. Syst. AES-29: 9-21

Moharir P S, Maru, V M, Singh R 1996 S-K-H algorithm for signal design. Electron. Lett. 32: $1648-1649$

Moharir P S, Maru V M, Singh R 1997 Bi-parental product algorithm for coded waveform design in radar. Sadhana 22: 589-599

Moharir P S, Maru M, Singh R 1998 Nonlinear geophysical signal analysis. Project completion report, CSIR-NGRI, Hyderabad 